\title{
Global Stability Analysis of Neural Networks with Constant Time Delay via Frobenius Norm
}

\author{
N. Mohamed Thoiyab, ${ }^{1}$ P. Muruganantham, ${ }^{1}$ Grienggrai Rajchakit ${ }^{(D)}{ }^{2}$ \\ Nallappan Gunasekaran, ${ }^{3}$ Bundit Unyong, ${ }^{4}$ Usa Humphries $\mathbb{D}^{5},{ }^{5}$ \\ Pramet Kaewmesri, ${ }^{5}$ and Chee Peng Lim ${ }^{6}$ \\ ${ }^{1}$ Department of Mathematics, Jamal Mohamed College, Affiliated to Bharathidasan University, Tiruchirappalli 620020, \\ Tamilnadu, India \\ ${ }^{2}$ Department of Mathematics, Faculty of Science, Maejo University, Sansai 50290, Chiang Mai, Thailand \\ ${ }^{3}$ Department of Mathematical Sciences, Shibaura Institute of Technology, Saitama 337-8570, Japan \\ ${ }^{4}$ Department of Mathematics, Faculty of Science and Technology, Phuket Rajabhat University, Phuket 83000, Thailand \\ ${ }^{5}$ Department of Mathematics, Faculty of Science, King Mongkut's University of Technology Thonburi (KMUTT), \\ 126 Pracha-Uthit Road, Bang Mod, Thung Khru 10140, Thailand \\ ${ }^{6}$ Institute for Intelligent Systems Research and Innovation, Deakin University, Waurn Ponds, VIC 3216, Australia
}

Correspondence should be addressed to Usa Humphries; usa.wan@kmutt.ac.th

Received 2 June 2020; Revised 27 July 2020; Accepted 12 August 2020; Published 12 October 2020

Academic Editor: Yuming Chen

Copyright (C) 2020 N. Mohamed Thoiyab et al. This is an open access article distributed under the Creative Commons Attribution License, which permits unrestricted use, distribution, and reproduction in any medium, provided the original work is properly cited.

\begin{abstract}
This paper deals with the global asymptotic robust stability (GARS) of neural networks (NNs) with constant time delay via Frobenius norm. The Frobenius norm result has been utilized to find a new sufficient condition for the existence, uniqueness, and GARS of equilibrium point of the NNs. Some suitable Lyapunov functional and the slope bounded functions have been employed to find the new sufficient condition for GARS of NNs. Finally, we give some comparative study of numerical examples for explaining the advantageous of the proposed result along with the existing GARS results in terms of network parameters.
\end{abstract}

\section{Introduction}

Neural networks (NNs) operate on principles similar to the human nervous system. It has a huge number of processors. These types of processors operate in parallel and are organized in layers. The initiating layer takes raw input, similar to the raw information received by humans. All subsequent layers receive input from the layer before it. Again, pass the output to the next layer. Finally, the end layer sends the final output. Most nodes are interconnected in layers.

NNs have been studied by many of the researchers because of their applications in different fields. The modern technology is mostly based on computational models which are known as artificial neural networks (ANNs). Nowadays artificial intelligence plays the most important role in electrical and electronics world. ANNs are the backbone of this artificial intelligence. In recent years, the role of ANNs has been developed due to their applications in various disciplines. The machine learning uses the distinct variety of NNs such as Feedforward NNs-artificial neuron, radial basis function NNs, multilayer perceptron, convolutional NNs, recurrent neural networks (RNNs), modular NNs, and sequence-to-sequence models. Moreover, NNs have wide applications in engineering areas [1-6] such as radar systems, signal classification, 3D reconstruction, face identification, object recognition, medical diagnosis, visualization, machine translation, combinatorial optimization, and signal processing. Also they may have great applications in nonengineering areas such as sales forecasting, risk management, and target marketing.

In NNs, time delay plays an important role in different areas such as video lip reading and speech recognition. Due 
to this delay parameter, the convergence of solution of the given system can be affected. The convergence of solution of neural system is to make the NNs to be stable. So, the concept of global stability analysis plays an important role in the convergence of solution of NNs. Also that the different kinds of stability analysis such as global asymptotic robust stability (GARS), exponential stability, and complete stability of NNs, have been studied by many researchers in [7-14]. These different types of stability results of the delayed NNs have been discussed based on the methods of Lyapunov stability theory, linear matrix inequalities, nonsmooth analysis, and M-matrix theory in the previous literature. Therefore, the GARS analysis of NNs under parameter uncertainties is the most important problem. Recently, it has been exclusively studied by many authors in [15-34].

From the motivation of the above concepts, GARS of NNs has been investigated in this paper. The objective of this paper is to obtain a new sufficient condition for the GARS of the equilibrium point of the delayed neural system using the Lyapunov stability theory and Frobenius norm under parameter uncertainties. The Frobenius norm is always an upper bound for the spectral norm $\left(\|\cdot\|_{2}\right)$. Moreover, the Frobenius norm is much easier to compute than the spectral norm. For calculating the Frobenius norm, it is not necessary to find the eigenvalues rather by using the traces (sum of the diagonals of a matrix). So, the calculation of finding eigenvalues is pretty difficult for higher dimensional matrices. Moreover, some matrices having real entries may give complex eigenvalues. By utilizing the Frobenius norm, we avoid such situations. Therefore, the Frobenius norm is important for calculating the upper bound of connection weight matrices. By utilizing the concept of homeomorphism, we find a new sufficient condition for the existence and uniqueness of the equilibrium point of NNs. Finally, we will give some comparative studies of numerical example to illustrate the effectiveness of our results for the NNs.

We apply the following notations for the norm of vectors and matrices: let $w=\left(w_{1}, w_{2}, \ldots, w_{n}\right)^{T} \in \mathbb{R}^{n}$. The most common vector norms $\|w\|_{1},\|w\|_{2}$, and $\|w\|_{\infty}$ which are defined as follows: $\|w\|_{1}=\sum_{i=1}^{n}\left|w_{i}\right|,\|w\|_{2}=\left(\sum_{i=1}^{n} w_{i}^{2}\right)^{1 / 2}$, and $\|w\|_{\infty}=\max _{1 \leq i \leq n}\left|w_{i}\right|$. Let $S=\left(s_{i j}\right)_{n \times n}$. The following are the definitions for $\|S\|_{1},\|S\|_{2},\|S\|_{\infty}$, and $\|S\|_{F} \cdot\|S\|_{1}=\max _{1 \leq j \leq n}$ $\sum_{i=1}^{n}\left|s_{i j}\right|,\|S\|_{2}=\left[\lambda_{\max }\left(S^{T} S\right)\right]^{1 / 2},\|S\|_{\infty}=\max _{1 \leq i \leq n} \sum_{j=1}^{n}\left|s_{i j}\right|$, and $\|S\|_{F}=\sqrt{\operatorname{tr}\left(S^{T} S\right)}=\sqrt{\operatorname{tr}\left(S S^{T}\right)}$. For any vector

$$
\left\{\begin{array}{l}
\mathscr{C}_{I}=\left\{\mathscr{C}=\operatorname{diag}\left(c_{i}\right): 0<\mathscr{C} \leq \mathscr{C} \leq \overline{\mathscr{C}},\right. \\
\mathscr{D}_{I}=\left\{\mathscr{D}=\left(d_{i j}\right): \underline{\mathscr{D}} \leq \mathscr{D} \leq \overline{\mathscr{D}},\right. \\
\mathscr{R}_{I}=\left\{\mathscr{R}=\left(r_{i j}\right): \underline{\mathscr{R}} \leq \mathscr{R} \leq \overline{\mathscr{R}},\right.
\end{array}\right.
$$

Assumption 1. (see [24]). The activation functions $f_{i}$ are assumed to be slope bounded; that is, there exist some positive constants $k_{i}$ such that the following conditions hold: $w=\left(w_{1}, w_{2}, \ldots, w_{n}\right)^{T},|w|$ is defined as $|w|=\left(\left|w_{1}\right|,\left|w_{2}\right|\right.$, $\left.\ldots,\left|w_{n}\right|\right)^{T}$. For any matrix $S=\left(s_{i j}\right)_{n \times n}$ with real entries $|S|$ will be defined as $|S|=\left(\left|s_{i j}\right|\right)_{n \times n}$. The minimum and maximum eigenvalues of $S$ are denoted by $\lambda_{\min }(S)$ and $\lambda_{\max }(S)$, respectively. $\operatorname{tr}(S)$ be the trace of the matrix $S$. That is, the sum of the diagonal values of the matrix $S$. If $S=\left(s_{i j}\right)_{n \times n}$ is a symmetric matrix and $w^{T} S w>0(\geq 0)$, for any real vector $w=\left(w_{1}, w_{2}, \ldots, w_{n}\right)^{T}$, then $S$ is said to be positive definite matrix (positive semidefinite matrix). Consider the two positive definite matrices $H=\left(h_{i j}\right)_{n \times n}$ and $S=\left(s_{i j}\right)_{n \times n}$. Then, $H<S$ implies that $w^{T} H w<w^{T} S w$ for any real vector $w=\left(w_{1}, w_{2}, \ldots, w_{n}\right)^{T}$.

\section{Problem Statement and Fundamentals}

In this paper, we consider the following delayed neural networks:

$$
\frac{\mathrm{d} w_{i}(t)}{\mathrm{d} t}=-c_{i} w_{i}(t)+\sum_{j=i}^{n} d_{i j} f_{j}\left(w_{j}(t)\right)+\sum_{j=i}^{n} r_{i j} f_{j}\left(w_{j}(t-\tau)\right)+J_{i},
$$

where $i=1,2, \ldots, n$ and $n$ denotes the total neurons. $w_{i}(t)$ denotes the $i^{\text {th }}$ neuron state of the vector at time $t$. $c_{i}$ represents the rate of charge for the $i^{\text {th }}$ neuron. $r_{i j}$ and $d_{i j}$ are the connection weight matrices with and without time delay, respectively. $f_{j}(\cdot)$ denotes the activation functions at time $t$ and $t-\tau$. Here $\tau$ denotes the constant time delay. $J_{i}$ represents the vector with constant input between the neurons.

The matrix vector form of equation (1) is as follows:

$$
\dot{w}(t)=-\mathscr{C} w(t)+\mathscr{D} f(w(t))+\mathscr{R}(f w(t-\tau)+J),
$$

where $\quad \mathscr{C}=\operatorname{diag}\left(c_{i}>0\right), \quad w(t)=\left[w_{1}(t), w_{2}(t), \ldots, w_{n}\right.$ $(t)]^{T} \in \mathbb{R}^{n}, \mathscr{R}=\left(r_{i j}\right) \in \mathbb{R}^{n \times n}, \mathscr{D}=\left(d_{i j}\right) \in \mathbb{R}^{n \times n}, f(w(t))=$ $\left[f_{1}\left(w_{1}(t)\right), f_{2}\left(w_{2}(t)\right), \ldots, f_{n}\left(w_{n}(t)\right)\right]^{T} \in \mathbb{R}^{n}, \quad$ and $f(w(t-\tau))=\left[f_{1}\left(w_{1}(t-\tau)\right), f_{2}\left(w_{2}(t-\tau)\right), \ldots, f_{n}\left(w_{n}\right.\right.$ $(t-\tau))]]^{T} \in \mathbb{R}^{n}, \quad J=\left[J_{1}, J_{2}, \ldots, J_{n}\right]^{T} \in \mathbb{R}^{n}$. The initial condition is $w(t)=\phi(t) \in C\left([-\tau, 0], \mathbb{R}^{n}\right)$, and $C([-\tau$, $\left.0], \mathbb{R}^{n}\right)$ denotes all continuous functions from $[-\tau, 0]$ to $\mathbb{R}^{n}$.

The most common approach for handling the delayed neural system is to make the connection weight matrices $\mathscr{D}=\left(d_{i j}\right)_{n \times n}$ and $\mathscr{R}=\left(r_{i j}\right)_{n \times n}$, and the matrix $\mathscr{C}=\operatorname{diag}\left(c_{i}>0\right)$ in an interval as follows:

$$
\begin{aligned}
& \text { ie., } \left.0<\underline{c}_{i} \leq c_{i} \leq \bar{c}_{i}, i=1,2, \ldots, n\right\}, \\
& \text { ie., } \left.\underline{d}_{i j} \leq d_{i j} \leq \bar{d}_{i j}, i, j=1,2, \ldots, n\right\}, \\
& \text { ie., } \left.\underline{r}_{i j} \leq r_{i j} \leq \bar{r}_{i j}, i, j=1,2, \ldots, n\right\} \text {. }
\end{aligned}
$$

$$
0 \leq \frac{f_{i}(w)-f_{i}(v)}{w-v} \leq k_{i}, \quad \forall w, v \in \mathbb{R}, w \neq v, i=1,2, \ldots, n .
$$


This class of functions will be denoted by $f \in k$. The functions of this class do not require to be bounded, differentiable, and monotonically increasing.
Lemma 1 (see [29]). Let $w(t)=\left[w_{1}(t), w_{2}(t), \ldots\right.$, $\left.w_{n}(t)\right]^{T} \in \mathbb{R}^{n}$. If $\mathscr{D} \in \mathscr{D}_{I}$ be the matrix defined as in equation (3), then for any positive diagonal matrix and a nonnegative diagonal matrix, the following inequality holds:

$$
w^{T}\left(\mathscr{M} \mathscr{D}+\mathscr{D}^{T} \mathscr{M}\right) w \leq w^{T}\left(\mathscr{M}\left(\mathscr{D}^{*}-\Upsilon\right)+\left(\mathscr{D}^{*}-\Upsilon\right)^{T} \mathscr{M}+\left\|\mathscr{M}\left(\mathscr{D}_{*}+\Upsilon\right)+\left(\mathscr{D}_{*}+\Upsilon\right)^{T} \mathscr{M}\right\|_{2} I\right) w,
$$

where $\mathscr{D}^{*}=(1 / 2(\overline{\mathscr{D}}+\underline{\mathscr{D}}))$ and $\mathscr{D}_{*}=(1 / 2(\overline{\mathscr{D}}-\underline{\mathscr{D}}))$.

Lemma 2 (see [24]). If $G(x) \in C^{0}\left(C^{0}\right.$ means that the set of continuous functions on $\mathbb{R}^{n}$ ) satisfies the following conditions, then $G(x)$ is a homeomorphism on $\mathbb{R}^{n}$ :

(i) $G(x)$ is an injective mapping on $\mathbb{R}^{n}$

(ii) $\|G(x)\| \longrightarrow \infty$ as $\|x\| \longrightarrow \infty$

\section{Existence and Uniqueness of Equilibrium Point}

This section will focus on the new sufficient condition for the existence of equilibrium point of our model (2) which is unique. By using the Frobenius norm, we prove a new sufficient condition for existence of equilibrium point of our NNs model (2) which is unique.

Theorem 1. Suppose that $f \in k$ and there exist matrices $K=\operatorname{diag}\left(k_{i}>0\right), \mathscr{M}=\operatorname{diag}\left(m_{i}>0\right)$, and $\Upsilon=\operatorname{diag}\left(\gamma_{i} \geq 0\right)$ such that

$$
\begin{aligned}
\Omega_{1}= & 2 \underline{\mathscr{C}} \mathscr{M} K^{-1}-\left(\mathscr{M}\left(\mathscr{D}^{*}-\Upsilon\right)+\left(\mathscr{D}^{*}-\Upsilon\right)^{T} \mathscr{M}+\left\|\left(\mathscr{M}\left(\mathscr{D}_{*}+\Upsilon\right)+\left(\mathscr{D}_{*}+\Upsilon\right)^{T} \mathscr{M}\right)\right\|_{2} I\right) \\
& -2\left(\mathscr{M}_{\max }\|\widehat{\mathscr{R}}\|_{F}\right) I>0,
\end{aligned}
$$

where $\mathscr{M}_{\text {max }}=\operatorname{maximum}\left(m_{i}\right), \mathscr{D}^{*}=1 / 2(\overline{\mathscr{D}}+\underline{\mathscr{D}}), \mathscr{D}_{*}=$ $1 / 2(\overline{\mathscr{D}}-\mathscr{D})$, and $\widehat{\mathscr{R}}=\left(\widehat{r}_{i j}\right)$ with $\widehat{r}_{i j}=\max \left(\left|\underline{r}_{i j}\right|,\left|\bar{r}_{i j}\right|\right)$. Then, for each constant vector $J$, the neural network model (2) satisfying (3) has a unique equilibrium point.

Proof. Define the following map:

$$
G(w)=-\mathscr{C} w+\mathscr{D} f(w)+\mathscr{R} f(w)+J .
$$

$G(w)=0$ is equivalent to $\dot{w}=0$. Here every solution of $G(w)=0$ is an equilibrium point of system (2). For proving this theorem, it is enough to prove that $G(w)$ is a homeomorphism on $\mathbb{R}^{n}$.

Let $w, v \in \mathbb{R}^{n}$ be the two vectors such that $w \neq v$.

Then,

$$
\begin{aligned}
G(w)-G(v)= & -\mathscr{C}(w-v)+\mathscr{D}(f(w)-f(v)) \\
& +\mathscr{R}(f(w)-f(v)) .
\end{aligned}
$$

If $f \in k, w \neq v$ then, $f(w)-f(v)=0$ or $f(w)-f(v) \neq 0$. First, let us consider $w \neq v$ and $f(w)-f(v)=0$. From equation (8), we have

$$
G(w)-G(v)=-\mathscr{C}(w-v) .
$$

Since $w \neq v$ and $\mathscr{C}=\operatorname{diag}\left(c_{i}>0\right)$. Therefore, from equation (9), $G(w) \neq G(v)$. Next, let us consider $w \neq v$ and $f(w)-f(v) \neq 0$ and multiply (9) by $2(f(w)-f(v))^{T} \mathscr{M}$; we get

$$
\begin{aligned}
2(f(w)-f(v))^{T} \mathscr{M}(G(w)-G(v))= & -2(f(w)-f(v))^{T} \mathscr{M} \mathscr{C}(w-v)+2(f(w)-f(v))^{T} \\
\mathscr{M} \mathscr{D}(f(w)-f(v))+2(f(w)-f(v))^{T} \mathscr{M} \mathscr{R}(f(w)-f(v))= & -2(f(w)-f(v))^{T} \mathscr{M} \mathscr{C}(w-v) \\
& +(f(w)-f(v))^{T}\left(\mathscr{M} \mathscr{D}+\mathscr{D}^{T} \mathscr{M}\right)(f(w)-f(v)) \\
& +2(f(w)-f(v))^{T} \mathscr{M} \mathscr{R}(f(w)-f(v)) .
\end{aligned}
$$


Then, we get

$$
\begin{aligned}
2(f(w)-f(v))^{T} \mathscr{M} \mathscr{C}(w-v)= & 2 \sum_{i=1}^{n} m_{i} c_{i}\left(f_{i}\left(w_{i}\right)-f_{i}\left(v_{i}\right)\right)\left(w_{i}-v_{i}\right) \\
\geq & 2 \sum_{i=1}^{n} \frac{m_{i} \underline{c}_{i}}{k_{i}}\left(f_{i}\left(w_{i}\right)-f_{i}\left(v_{i}\right)\right)=2(f(w)-f(v))^{T} \underline{C} \mathscr{M} K^{-1}(f(w)-f(v)), \\
& -2(f(w)-f(v))^{T} \mathscr{M} \mathscr{C}(w-v) \leq-2(f(w)-f(v))^{T} \underline{\mathscr{C}} \mathscr{M} K^{-1}(f(w)-f(v)) .
\end{aligned}
$$

From Lemma 1, we get

$$
\begin{aligned}
& (f(w)-f(v))^{T}\left(\mathscr{M} \mathscr{D}+\mathscr{D}^{T} \mathscr{M}\right)(f(w)-f(v)) \leq(f(w)-f(v))^{T} \\
& \cdot\left(\mathscr{M}\left(\mathscr{D}^{*}-\Upsilon\right)+\left(\mathscr{D}^{*}-\Upsilon\right)^{T} \mathscr{M}+\left\|\mathscr{M}\left(\mathscr{D}_{*}+\Upsilon\right)+\left(\mathscr{D}_{*}+\Upsilon\right)^{T} \mathscr{M}\right\|_{2} I\right) \\
& \cdot(f(w)-f(v)) \text {. } \\
& 2(f(w)-f(v))^{T} \mathscr{M} \mathscr{R}(f(w)-f(v))=\sum_{i=1}^{n} \sum_{j=1}^{n} 2 m_{i} r_{i j}\left(f_{i}\left(w_{i}\right)-f_{i}\left(v_{i}\right)\right)\left(f_{j}\left(w_{j}\right)-f_{j}\left(v_{j}\right)\right) \\
& \leq 2 \mathscr{M}_{\max } \sum_{i=1}^{n} \sum_{j=1}^{n} \widehat{r}_{i j}\left(f_{i}\left(w_{i}\right)-f_{i}\left(v_{i}\right)\right)\left(f_{j}\left(w_{j}\right)-f_{j}\left(v_{j}\right)\right) \\
& \leq 2 \mathscr{M}_{\max }\|\widehat{\mathscr{R}}\|_{F} \|(f(w)-f(v))^{T}(f(w)-f(v)) \text {. }
\end{aligned}
$$

Applying the results (11)-(13) in (10), we get

$$
\begin{aligned}
2(f(w)-f(v))^{T} \mathscr{M}(G(w)-G(v)) \leq & -2(f(w)-f(v))^{T} \\
& \underline{\mathscr{C}} \mathscr{M} K^{-1}(f(w)-f(v))+(f(w)-f(v))^{T} \\
& \left(\mathscr{M}\left(\mathscr{D}^{*}-\Upsilon\right)+\left(\mathscr{D}^{*}-\Upsilon\right)^{T} \mathscr{M}+\left\|\mathscr{M}\left(\mathscr{D}_{*}+\Upsilon\right)+\left(\mathscr{D}_{*}+\Upsilon\right)^{T} \mathscr{M}\right\|_{2} I\right) \\
& (f(w)-f(v))+2 \mathscr{M}_{\max }\|\widehat{\mathscr{R}}\|_{F}(f(w)-f(v))^{T}(f(w)-f(v)) \\
= & -(f(w)-f(v))^{T} \Omega_{1}(f(w)-f(v)) .
\end{aligned}
$$

Given that $\Omega_{1}>0$, we have

$$
2(f(w)-f(v))^{T} \mathscr{M}(G(w)-G(v)) \leq-\lambda_{\min }\left(\Omega_{1}\right)\|(f(w)-f(v))\|_{2}^{2},
$$

where $\lambda_{\min }\left(\Omega_{1}\right)$ is the smallest eigen value of the positive definite matrix $\Omega_{1}$. If $f(w)-f(v) \neq 0$ and $\lambda_{\min }\left(\Omega_{1}\right)>0$, then it follows from (14) that $2(f(w)-f(v))^{T} \mathscr{M}(G(w)$ $-G(v))<0$.
Therefore, $G(w) \neq G(v)$ for all $w \neq v$. Hence $G$ is injective on $\mathbb{R}^{n}$. Now, we prove that $\|G(w)\| \longrightarrow \infty$ as $\|w\| \longrightarrow \infty$. For this, let $v=0$ in (15) which implies that 


$$
\begin{gathered}
2(f(w)-f(0))^{T} \mathscr{M}(G(w)-G(0)) \leq-\lambda_{\min }\left(\Omega_{1}\right)\|(f(w)-f(0))\|_{2}^{2}, \\
\left|2(f(w)-f(0))^{T} \mathscr{M}(G(w)-G(0))\right| \geq \lambda_{\min }\left(\Omega_{1}\right)\|(f(w)-f(0))\|_{2}^{2} .
\end{gathered}
$$

From the above inequality, we write

$$
\begin{gathered}
2\|\mathscr{M}\|_{\infty}\|f(w)-f(0)\|_{\infty}\|G(w)-G(0)\|_{1} \\
>\lambda_{\min }\left(\Omega_{1}\right)\|(f(w)-f(0))\|_{2}^{2} .
\end{gathered}
$$

By applying the properties of norm for the above inequalities, we have

$$
\begin{aligned}
\|f(w)-f(0)\|_{\infty} & \leq\|f(w)-f(0)\|_{2}, \\
\|G(w)-G(0)\|_{1} & \leq\|G(w)\|_{1}+\|G(0)\|_{1}, \\
\|f(w)-f(0)\|_{2} & \geq\|f(w)\|_{2}-\|f(0)\|_{2} .
\end{aligned}
$$

Using the above inequalities in (20), we have the following inequality:

$$
\|G(w)\|_{1}>\frac{\lambda_{\min }\left(\Omega_{1}\right)\|f(w)\|_{2}-\lambda_{\min }\left(\Omega_{1}\right)\|f(0)\|_{2}-2\|\mathscr{M}\|_{\infty}\|G(0)\|_{1}}{2\|\mathscr{M}\|_{\infty}},
$$

where $\|f(0)\|_{2},\|G(0)\|_{1}$, and $\|\mathscr{M}\|_{\infty}$ are finite. Moreover, $\|G(w)\| \longrightarrow \infty$ as $\|f(w)\| \longrightarrow \infty$ or equivalently $\|G(w)\| \longrightarrow \infty$ as $\|w\| \longrightarrow \infty$. Therefore, from the result of Lemma 2, for each constant vector $J$, the neural networks model (2) has a unique equilibrium point. Hence, the proof.

\section{Global Stability Analysis}

In this section, we prove that the obtained sufficient conditions for the existence and uniqueness of the equilibrium point in the previous Theorem 1 will also give the sufficient conditions for the GARS of the neural system (2). Furthermore, we denote the equilibrium point of 1 by $w^{*}$ and use some proper transformation say $y_{i}(\cdot)=w_{i}(\cdot)$ $-w_{i}^{*}, i=1,2, \ldots, n$. After giving such transformation, the network model (1) can be put in the following form:

$$
\dot{y}_{i}(t)=-c_{i} y_{i}(t)+\sum_{j=1}^{n} d_{i j} g_{j}\left(y_{j}(t)\right)+\sum_{j=1}^{n} r_{i j} g_{j}\left(y_{j}(t-\tau)\right),
$$

where $g_{i}\left(y_{i}(\cdot)\right)=f_{i}\left(y_{i}(\cdot)+w_{i}^{*}\right)-f_{i}\left(w_{i}^{*}\right), i=1,2, \ldots, n$ Moreover, Assumption 1 holds for the function $g$, i.e., $f \in k$ gives that $g \in k$ with $g_{i}(0)=0, i=1,2, \ldots, n$. By using this transformation, the equilibrium point $w^{*}$ of 2 is shifted to the origin of (20).

Now, our focus is to show that GARS for the origin of the transformed model (20) instead of focusing the GARS for $w^{*}$.

The matrix form of (20) is as follows:

$$
\dot{y}(t)=-\mathscr{C} y(t)+\mathscr{D} g(y(t))+\mathscr{R} g(y(t-\tau)),
$$

where $y(t)=\left(y_{1}(t), y_{2}(t), \ldots, y_{n}(t)\right)^{T} \in \mathbb{R}^{n}$ is the new state vector, $g(y(t))=\left(g_{1}\left(y_{1}(t)\right), g_{2}\left(y_{2}(t)\right), \ldots, g_{n}\right.$ $\left.\left(y_{n}(t)\right)\right)^{T} \in \mathbb{R}^{n}, \quad$ and $g(y(t-\tau))=\left(g_{1}\left(y_{1}(t-\tau)\right), g_{2}\right.$ $\left.\left(y_{2}(t-\tau)\right), \ldots, g_{n}\left(y_{n}(t-\tau)\right)\right)^{T} \in \mathbb{R}^{n}$.

Theorem 2. Suppose that $g \in k$ and there exist matrices $K=\operatorname{diag}\left(k_{i}>0\right), \mathscr{M}=\operatorname{diag}\left(m_{i}>0\right)$, and $\Upsilon=\operatorname{diag}\left(\gamma_{i} \geq 0\right)$, such that

$$
\begin{aligned}
\Omega_{1}= & 2 \underline{\mathscr{C}} \mathscr{M} K^{-1}-\left(\mathscr{M}\left(\mathscr{D}^{*}-\Upsilon\right)+\left(\mathscr{D}^{*}-\Upsilon\right)^{T} \mathscr{M}+\left\|\mathscr{M}\left(\mathscr{D}_{*}+\Upsilon\right)+\left(\mathscr{D}_{*}+\Upsilon\right)^{T} \mathscr{M}\right\|_{2} I\right) \\
& -2\left(\mathscr{M}_{\max }\|\widehat{\mathscr{R}}\|_{F}\right) I>0,
\end{aligned}
$$

where $\mathscr{M}_{\text {max }}=\operatorname{maximum}\left(m_{i}\right), \mathscr{D}^{*}=1 / 2(\overline{\mathscr{D}}+\mathscr{D}), \mathscr{D}_{*}=1 / 2$ $(\overline{\mathscr{D}}-\underline{\mathscr{D}})$, and $\widehat{\mathscr{R}}=\left(\widehat{r}_{i j}\right)$ with $\widehat{r}_{i j}=\max \left(\left|\underline{r}_{i j}\right|,\left|\bar{r}_{i j}\right|\right)$. Then, origin of the neural network model (21) satisfying the network parameters 3 is globally asymptotically robust stable.
Proof. Consider the following positive definite Lyapunov functional:

$$
V(y(t))=y^{T}(t) y(t)+2 \delta \sum_{i=1}^{n} \int_{0}^{y_{i}(t)} m_{i} g_{i}(\xi) \mathrm{d} \xi+(\delta \mu+\eta) \sum_{i=1}^{n} \int_{t-\tau_{i}}^{t} g_{i}^{2}\left(y_{i}(\zeta)\right) \mathrm{d} \zeta
$$


where $m_{i}, \delta, \eta$, and $\mu$ are the positive constants which will be determined later. The following equation is the time derivative of equation (23) along the trajectories of the model (21):

$$
\begin{aligned}
\dot{V}(y(t))= & -2 \delta g^{T}(y(t)) \mathscr{M} \mathscr{C} y(t)+2 \delta g^{T}(y(t)) \mathscr{M} \mathscr{D} g(y(t)) \\
& +2 \delta g^{T}(y(t)) \mathscr{M} \mathscr{R} g(y(t-\tau))+2 y^{T}(t) \mathscr{R} g(y(t-\tau)) \\
& -2 y^{T}(t) \mathscr{C} y(t)+2 y^{T}(t) \mathscr{D} g(y(t))+\delta \mu\|g(y(t))\|_{2}^{2}-\delta \mu\|g(y(t-\tau))\|_{2}^{2} \\
& +\eta\|g(y(t))\|_{2}^{2}-\eta\|g(y(t-\tau))\|_{2}^{2} .
\end{aligned}
$$

Also,

$$
\begin{aligned}
-y^{T}(t) \mathscr{C} y(t)+2 y^{T}(t) \mathscr{D} g(y(t)) & \leq g^{T}(y(t)) \mathscr{D}^{T} \mathscr{C}^{-1} \mathscr{D} g(y(t)) \\
& \leq\|\mathscr{D}\|_{2}^{2}\left\|\mathscr{C}^{-1}\right\|_{2}\|g(y(t))\|_{2}^{2}, \\
-y^{T}(t) \mathscr{C} y(t)+2 y^{T}(t) \mathscr{R} g(y(t-\tau)) & \leq g^{T}(y(t-\tau)) \mathscr{R}^{T} \mathscr{C}^{-1} \mathscr{R} g(y(t-\tau)) \\
& \leq\|\mathscr{R}\|_{2}^{2}\left\|\mathscr{C}^{-1}\right\|_{2}\|g(y(t-\tau))\|_{2}^{2}, \\
2 \delta g^{T}(y(t)) \mathscr{M} \mathscr{R} g(y(t-\tau)) & \leq 2 \delta \mathscr{M}_{\max }\|\mathscr{R}\|_{2}\|g(y(t))\|_{2}\|g(y(t-\tau))\|_{2} \\
& \leq \delta \mathscr{M}_{\max }\|\mathscr{R}\|_{2}\|g(y(t))\|_{2}^{2}+\delta \mathscr{M}_{\max }\|\mathscr{R}\|_{2}\|g(y(t-\tau))\|_{2}^{2} \\
& \leq \delta \mathscr{M}_{\max }\|\widehat{\mathscr{R}}\|_{F} g(y(t))\left\|_{2}^{2}+\delta \mathscr{M}_{\max }\right\| \widehat{\mathscr{R}}\left\|_{F} g(y(t-\tau))\right\|_{2}^{2}, \\
-2 \delta g^{T}(y(t)) \mathscr{M} \mathscr{C} y(t) & \leq-2 \delta g^{T}(y(t)) \mathscr{M}_{\underline{C}} K^{-1} g(y(t)) .
\end{aligned}
$$

By applying equations (25)-(28) in (24), we have

$$
\begin{aligned}
\dot{V}(y(t)) \leq & \|\mathscr{D}\|_{2}^{2}\left\|\mathscr{C}^{-1}\right\|_{2}\|g(y(t))\|_{2}^{2}+\|\mathscr{R}\|_{2}^{2}\left\|\mathscr{C}^{-1}\right\|_{2}\|g(y(t-\tau))\|_{2}^{2}-2 \delta g^{T}(y(t)) \mathscr{M}_{\mathscr{C}} K^{-1} g(y(t)) \\
& +\delta g^{T}(y(t))\left(\mathscr{M} \mathscr{D}+\mathscr{D}^{T} \mathscr{M}\right) g(y(t))+\delta \mathscr{M}_{\max }\|\widehat{\mathscr{R}}\|_{F}\|g(y(t))\|_{2}^{2}+\delta \mathscr{M}_{\max }\|\widehat{\mathscr{R}}\|_{F}\|g(y(t-\tau))\|_{2}^{2} \\
& +\delta \mu\|g(y(t))\|_{2}^{2}-\delta \mu\|g(y(t-\tau))\|_{2}^{2}+\eta\|g(y(t))\|_{2}^{2}-\eta\|g(y(t-\tau))\|_{2}^{2} .
\end{aligned}
$$

Since $\|\mathscr{D}\|_{2} \leq\|\widehat{\mathscr{D}}\|_{F},\|\mathscr{R}\|_{2} \leq\|\widehat{\mathscr{R}}\|_{F}$, and $\left\|\mathscr{C}^{-1}\right\|_{2} \leq\left\|\underline{\mathscr{C}}^{-1}\right\|_{2}$. Therefore,

$$
\begin{aligned}
\dot{V}(y(t)) \leq & \|\widehat{\mathscr{D}}\|_{F}^{2}\|\underline{\mathscr{C}}\|_{2}^{-1}\|g(y(t))\|_{2}^{2}+\|\widehat{\mathscr{R}}\|_{F}^{2}\left\|\underline{\mathscr{C}}^{-1}\right\|_{2}\|g(y(t-\tau))\|_{2}^{2}-2 \delta g^{T}(y(t)) \mathscr{M}_{\underline{\mathscr{C}}} K^{-1} g(y(t)) \\
& +\delta g^{T}(y(t))\left(\mathscr{M} \mathscr{D}+\mathscr{D}^{T} \mathscr{M}\right) g(y(t))+\delta \mathscr{M}_{\max }\|\widehat{\mathscr{R}}\|_{F}\|g(y(t))\|_{2}^{2}+\delta \mathscr{M}_{\max }\|\widehat{\mathscr{R}}\|_{F}\|g(y(t-\tau))\|_{2}^{2} \\
& +\delta \mu\|g(y(t))\|_{2}^{2}-\delta \mu\|g(y(t-\tau))\|_{2}^{2}+\eta\|g(y(t))\|_{2}^{2}-\eta\|g(y(t-\tau))\|_{2}^{2} .
\end{aligned}
$$


By taking $\eta=\|\hat{\mathscr{R}}\|_{F}^{2}\left\|\mathscr{C}^{-1}\right\|_{2}$ and $\mu=\mathscr{M}_{\max }\|\widehat{\mathscr{R}}\|_{F}, \dot{V}(y(t))$ can be written in the following form:

$$
\begin{aligned}
\dot{V}(y(t)) \leq & \left(\|\widehat{\mathscr{D}}\|_{F}^{2}+\|\widehat{\mathscr{R}}\|_{F}^{2}\right)\left\|_{\mathscr{\mathscr { C }}}^{-1}\right\|_{2}\|g(y(t))\|_{2}^{2}-2 \delta g^{T}(y(t)) \mathscr{M}_{\mathscr{C}} K^{-1} g(y(t)) \\
& +\delta g^{T}(y(t))\left(\mathscr{M} \mathscr{D}+\mathscr{D}^{T} \mathscr{M}\right) g(y(t))+2 \delta \mathscr{M}_{\max }\|\widehat{\mathscr{R}}\|_{F}\|g(y(t))\|_{2}^{2} .
\end{aligned}
$$

Using the result of Lemma 2, we write

$$
\begin{aligned}
g^{T}(y(t))\left(\mathscr{M} \mathscr{D}+\mathscr{D}^{T} \mathscr{M}\right) g(y(t)) \leq & g^{T}(y(t)) \\
& \left(\mathscr{M}\left(\mathscr{D}^{*}-\Upsilon\right)+\left(\mathscr{D}^{*}-\Upsilon\right)^{T} \mathscr{M}+\left\|\mathscr{M}\left(\mathscr{D}_{*}+\Upsilon\right)+\left(\mathscr{D}_{*}+\Upsilon\right)^{T} \mathscr{M}\right\|_{2} I\right) g(y(t)) .
\end{aligned}
$$

Then, $\dot{V}(y(t))$ becomes

$$
\begin{aligned}
\dot{V}(y(t)) \leq & \left(\|\widehat{\mathscr{D}}\|_{F}^{2}+\|\widehat{\mathscr{R}}\|_{F}^{2}\right)\|\underline{\mathscr{C}}\|_{2}\|g(y(t))\|_{2}^{2}-2 \delta g^{T}(y(t)) \mathscr{M} \underline{\mathscr{C}} K^{-1} g(y(t)) \\
& +\delta g^{T}(y(t))\left(\mathscr{M}\left(\mathscr{D}^{*}-\Upsilon\right)+\left(\mathscr{D}^{*}-\Upsilon\right)^{T} \mathscr{M}+\left\|\mathscr{M}\left(\mathscr{D}_{*}+\Upsilon\right)+\left(\mathscr{D}_{*}+\Upsilon\right)^{T} \mathscr{M}\right\|_{2} I\right) g(y(t)) \\
& +2 \delta \mathscr{M}_{\max }\|\widehat{\mathscr{R}}\|_{F}\|g(y(t))\|_{2}^{2}=\left(\|\widehat{\mathscr{D}}\|_{F}^{2}+\|\widehat{\mathscr{R}}\|_{F}^{2}\right)\|\underline{\mathscr{C}}\|_{2}\|g(y(t))\|_{2}^{2}-\delta g^{T}(y(t)) \Omega_{1} g(y(t)) .
\end{aligned}
$$

Since $\Omega_{1}>0$, it follows from (33) that

$$
\dot{V}(y(t)) \leq\left(\|\widehat{\mathscr{D}}\|_{F}^{2}+\|\widehat{\mathscr{R}}\|_{F}^{2}\right)\|\underline{\mathscr{C}}\|_{2}^{-1}\|g(y(t))\|_{2}^{2}-\delta \lambda_{\min }\left(\Omega_{1}\right)\|g(y(t))\|_{2}^{2}
$$

If we take $\delta>\left(\|\widehat{\mathscr{D}}\|_{F}^{2}+\|\widehat{\mathscr{R}}\|_{F}^{2}\right)\left\|\mathscr{C}^{-1}\right\|_{2} / \lambda_{\text {min }}\left(\Omega_{1}\right)$, then it gives that $y(t) \neq 0$. Also if $g(y(t))=0$ and $y(t) \neq 0$, then follows that $\dot{V}(y(t))<0, \forall g(y(t)) \neq 0$ since $g(y(t)) \neq 0$

$$
\begin{aligned}
\dot{V}(y(t))= & -\eta g^{T}(y(t-\tau)) g(y(t-\tau))-\delta \mu g^{T}(y(t-\tau)) g(y(t-\tau)) \\
& -2 y^{T}(t) \mathscr{C} y(t)+2 y^{T}(t) \mathscr{R} g(t-\tau) \\
\leq & -2 y^{T}(t) \mathscr{C} y(t)+2 y^{T}(t) \mathscr{R} g(t-\tau)-\eta g^{T}(y(t-\tau)) g(y(t-\tau)) .
\end{aligned}
$$

Since $-y^{T}(t) \mathscr{C} y(t)+2 y^{T}(t) \mathscr{R} g(t-\tau)-\eta g^{T}(y(t-\tau))$ $g(y(t-\tau)) \leq 0$, then we have $\dot{V}(y(t))=-y^{T}(t) \mathscr{C} y(t)$. Therefore $\dot{V}(y(t))<0, \forall y(t) \neq 0$. Finally, let us assume that $g(y(t))=0$ and $y(t)=0$. Then, $\dot{V}(y(t))=-\eta g^{T}$ $(y(t-\tau)) g(y(t-\tau))-\delta \mu g^{T}(y(t-\tau)) g(y(t-\tau))$.

It is obvious that $\dot{V}(y(t))<0, \forall g(y(t-\tau)) \neq 0$. Hence, $\dot{V}(y(t))=0$ if and only if $y(t)=g(y(t))=g(y(t-\tau))=0$; otherwise, $\dot{V}(y(t))<0$. Moreover, $V(y(t))$ is radially unbounded provided $V(y(t)) \longrightarrow \infty$ as $\|y\| \longrightarrow \infty$. Hence, origin of system (21), or the equilibrium point of the neural system (2), is GARS.

\section{Comparisons with Numerical Examples}

In this section, we will compare our sufficient conditions of GARS with the previous existing results of GARS. For the comparison, the previous results of GARS are restated as follows. 
Theorem 3 (see [24]). Suppose that $g \in k$ and there exist matrices $K=\operatorname{diag}\left(k_{i}>0\right)$ and $\mathscr{M}=\operatorname{diag}\left(m_{i}>0\right)$ such that

$$
\Omega_{2}=2 \underline{\mathscr{C}} \mathscr{M} K^{-1}-\left(\mathscr{M} \mathscr{D}^{*}+\left(\mathscr{D}^{*}\right)^{T} \mathscr{M}+\left\|\mathscr{M} \mathscr{D}_{*}+\left(\mathscr{D}_{*}\right)^{T} \mathscr{M}\right\|_{2} I\right)-2\left(\|\mathscr{M}\|_{2}\|\widehat{\mathscr{R}}\|_{F}\right) I>0 .
$$

Then, origin of system (21) satisfying the network parameters 3 is globally asymptotically robust stable.

Theorem 4 (see [15]). Suppose that $g \in k$ and there exist matrices $K=\operatorname{diag}\left(k_{i}>0\right)$ and $\mathscr{M}=\operatorname{diag}\left(m_{i}>0\right)$ such that

$$
\Omega_{3}=2 \underline{\mathscr{C}} \mathscr{M} K^{-1}+\mathscr{Z}-2\left(\|\mathscr{M}\|_{2}\|\widehat{\mathscr{R}}\|_{F}\right) I>0 .
$$

Then origin of system (21) satisfying the network parameters 3 is globally asymptotically robust stable., where $\mathscr{Z}=$ $\left(z_{i j}\right)_{n \times n}$ with $z_{i i}=-2 m_{i} \bar{d}_{i i}$ and $z_{i j}=-\operatorname{maximum}\left(\mid m_{i} \bar{d}_{i j}+\right.$ $\left.m_{j} d_{j i}|,| m_{i} \underline{d}_{i j}+m_{j} \underline{d}_{j i} \mid\right)$, for $i \neq j$. Now we demonstrate the advantages of our result with some examples as follows.

Example 1. Consider the following network parameters of the neural network model 2:

$$
\begin{aligned}
& \underline{\mathscr{D}}=\left[\begin{array}{cccc}
1 & -1 & -1 & -1 \\
0 & 1 & 0 & -1 \\
-2 & -1 & 1 & -1 \\
-2 & -2 & 0 & 1
\end{array}\right], \\
& \overline{\mathscr{D}}=\left[\begin{array}{cccc}
1 & 1 & 1 & 1 \\
2 & 1 & 2 & 1 \\
0 & 1 & 1 & 1 \\
0 & 0 & 2 & 1
\end{array}\right], \\
& \underline{\mathscr{R}}=\left[\begin{array}{cccc}
-1 & 0 & -1 & 0 \\
0 & -1 & -1 & 1 \\
-1 & 0 & -1 & 1 \\
1 & -1 & 1 & -1
\end{array}\right], \\
& \overline{\mathscr{R}}=\left[\begin{array}{cccc}
1 & 1 & 0 & 1 \\
0 & 1 & -1 & 1 \\
1 & 0 & 1 & 1 \\
1 & 1 & 1 & -1
\end{array}\right] .
\end{aligned}
$$

Let $k_{1}=k_{2}=k_{3}=k_{4}=1 \quad$ and $\quad \underline{c}_{1}=\underline{c}_{2}=\underline{c}_{3}=\underline{c}_{4}=c$. From the above matrices, we get

$$
\mathscr{D}^{*}=\left[\begin{array}{cccc}
1 & 0 & 0 & 0 \\
1 & 1 & 1 & 0 \\
-1 & 0 & 1 & 0 \\
-1 & -1 & 1 & 1
\end{array}\right],
$$$$
\mathscr{D}_{*}=a\left[\begin{array}{llll}
0 & 1 & 1 & 1 \\
1 & 0 & 1 & 1 \\
1 & 1 & 0 & 1 \\
1 & 1 & 1 & 0
\end{array}\right] \text {, }
$$$$
\widehat{\mathscr{R}}=\left[\begin{array}{llll}
1 & 1 & 1 & 1 \\
1 & 1 & 1 & 1 \\
1 & 1 & 1 & 1 \\
1 & 1 & 1 & 1
\end{array}\right] \text {, }
$$

$$
\Upsilon=\left[\begin{array}{llll}
1 & 0 & 0 & 0 \\
0 & 1 & 0 & 0 \\
0 & 0 & 1 & 0 \\
0 & 0 & 0 & 1
\end{array}\right] \text {. }
$$

Using the above parameters, $\|\widehat{\mathscr{R}}\|_{F}=4$.

In this example, we compare our sufficient condition $\Omega_{1}$ with the result $\Omega_{3}$ by taking $\mathscr{M}$ as an identity matrix. Now, $\Omega_{1}$ and $\Omega_{3}$ are calculated as follows:

$$
\begin{aligned}
\Omega_{1} & =2 \underline{\mathscr{C}} \mathscr{M} K^{-1}-\left(\mathscr{M}\left(\mathscr{D}^{*}-\Upsilon\right)+\left(\mathscr{D}^{*}-\Upsilon\right)^{T} \mathscr{M}+\left\|\mathscr{M}\left(\mathscr{D}_{*}+\Upsilon\right)+\left(\mathscr{D}_{*}+\Upsilon\right)^{T} \mathscr{M}\right\|_{2} I\right) \\
-2\left(\mathscr{M}_{\max }\|\widehat{\mathscr{R}}\|_{F}\right) I & =\left[\begin{array}{cccc}
2 c-16 & -1 & 1 & 1 \\
-1 & 2 c-16 & -1 & 1 \\
1 & -1 & 2 c-16 & -1 \\
1 & 1 & -1 & 2 c-16
\end{array}\right] .
\end{aligned}
$$

$\Omega_{1}>0$, provided $c>9.12$. For the sufficient condition $\Omega_{1}>0$, system 2 will become GARS whenever $c>9.12$. For calculating $\Omega_{3}$, we need the matrix $\mathscr{Z}$, and it is calculated using the matrices $\mathscr{M}, \mathscr{D}$, and $\overline{\mathscr{D}}$. The matrix $\mathscr{Z}$ is given as follows: 


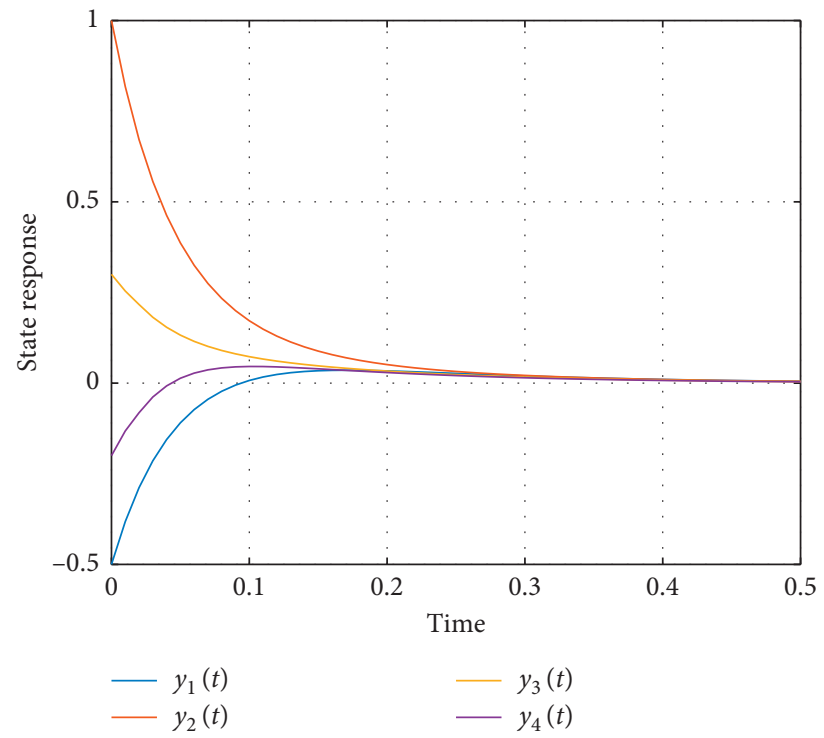

FIGURE 1: System solution for the initial state $y(0)=[-0.5,1,0.3,-0.2]$.

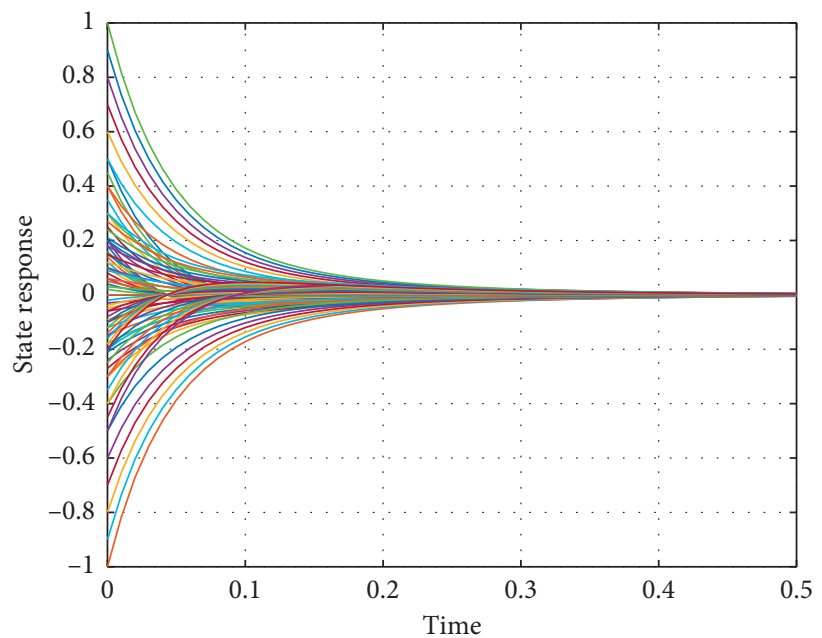

Figure 2: System solution for the different initial states.

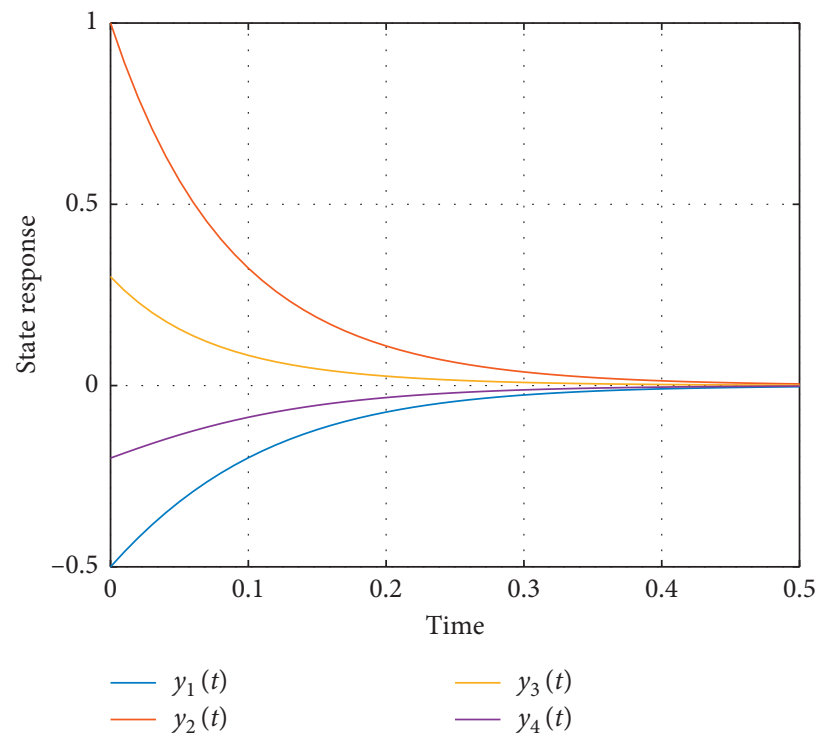

Figure 3: System solution for the initial state $y(0)=[-0.5,1,0.3,-0.2]$. 


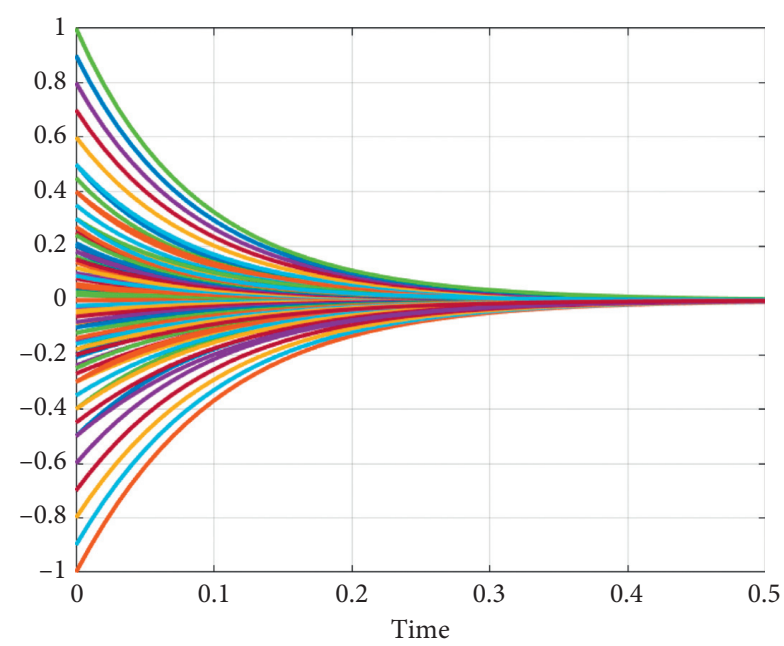

Figure 4: System solution for the different initial states.

$$
\begin{aligned}
\mathscr{Z} & =\left[\begin{array}{llll}
-2 & -3 & -3 & -3 \\
-3 & -2 & -3 & -3 \\
-3 & -3 & -2 & -3 \\
-3 & -3 & -3 & -2 .
\end{array}\right], \\
\Omega_{3} & =2 \underline{\mathscr{C}} \mathscr{M} K^{-1}-+\mathscr{Z}-2\left(\|\mathscr{M}\|_{2}\|\widehat{\mathscr{R}}\|_{F}\right) I \\
& =2\left[\begin{array}{cccc}
2 c-10 & -3 & -3 & -3 \\
-3 & 2 c-10 & -3 & -3 \\
-3 & -3 & 2 c-10 & -3 \\
-3 & -3 & -3 & 2 c-10
\end{array}\right] .
\end{aligned}
$$

$\Omega_{3}>0$, provided $c>9.5$. For the sufficient condition $\Omega_{3}>0$, system 2 will become GARS whenever $c>9.5$. Consider the fixed network parameters $\mathscr{C}=20 I, \mathscr{D}=$ $\overline{\mathscr{D}}$, and $\mathscr{R}=\overline{\mathscr{R}}, \tau$ is a constant, and the activation function $g(y(t))=\tanh (y(t))$. The state trajectories are depicted in Figures 1 and 2 under the initial state $y(0)=[-0.5$, $1,0.3,-0.2]$ and for the different initial states, respectively. The activation function $g(y(t))=\left(e^{-y^{2}(t)}-1 / e^{-y^{2}(t)}+1\right)$, and the state trajectories are depicted in Figures 3 and 4 under the initial state $y(0)=[-0.5,1,0.3,-0.2]$ and for the different initial states, respectively.

Remark 1. From the above Example $1, \Omega_{3}$ is valid for $c>9.5$. Moreover, our result $\Omega_{1}$ is valid in the range $9.12<c<9.5$, but $\Omega_{3}$ does not hold. Therefore, we conclude that $\Omega_{1}$ is less conservative than $\Omega_{3}$ for the network parameters of this example.

Example 2. Consider the neural network model 1 with the following network parameters:

$$
\begin{aligned}
& \underline{\mathscr{D}}=\left[\begin{array}{cccc}
-2 & -1 & -1 & -1 \\
-1 & 0 & -1 & -1 \\
-1 & -1 & 0 & -1 \\
-1 & -1 & -1 & 0
\end{array}\right], \\
& \overline{\mathscr{D}}=\left[\begin{array}{llll}
0 & 1 & 1 & 1 \\
1 & 0 & 1 & 1 \\
1 & 1 & 0 & 1 \\
1 & 1 & 1 & 0
\end{array}\right], \\
& \underline{\mathscr{R}}=\left[\begin{array}{cccc}
-2 & 0 & -1 & -2 \\
0 & -1 & -1 & -2 \\
-2 & -2 & -1 & 1 \\
1 & -2 & 1 & -1
\end{array}\right],
\end{aligned}
$$

$$
\overline{\mathscr{R}}=\left[\begin{array}{llll}
1 & 2 & 2 & 1 \\
2 & 2 & 2 & 1 \\
1 & 0 & 2 & 2 \\
2 & 1 & 2 & 2
\end{array}\right] .
$$

Let $k_{1}=k_{2}=k_{3}=k_{4}=1 \quad$ and $\quad \underline{c}_{1}=\underline{c}_{2}=\underline{c}_{3}=\underline{c}_{4}=c$. From the above matrices, we get

$$
\begin{aligned}
& \mathscr{D}^{*}=\left[\begin{array}{llll}
-1 & 0 & 0 & 0 \\
0 & 0 & 0 & 0 \\
0 & 0 & 0 & 0 \\
0 & 0 & 0 & 0
\end{array}\right], \\
& \mathscr{D}_{*}=a\left[\begin{array}{llll}
1 & 1 & 1 & 1 \\
1 & 0 & 1 & 1 \\
1 & 1 & 0 & 1 \\
1 & 1 & 1 & 0
\end{array}\right], \\
& \widehat{\mathscr{R}}=\left[\begin{array}{llll}
2 & 2 & 2 & 2 \\
2 & 2 & 2 & 2 \\
2 & 2 & 2 & 2 \\
2 & 2 & 2 & 2
\end{array}\right], \\
& \Upsilon= \\
& \left.0 \begin{array}{llll}
0 & 0 & 0 & 0 \\
0 & 1 & 0 & 0 \\
0 & 0 & 1 & 0 \\
0 & 0 & 0 & 1
\end{array}\right] .
\end{aligned}
$$

Using the above parameters, $\|\widehat{\mathscr{R}}\|_{F}=8$. In this example, we compare our sufficient condition $\Omega_{1}$ with the result $\Omega_{2}$ by taking $\mathscr{M}$ as an identity matrix. Now, $\Omega_{1}$ and $\Omega_{2}$ are calculated as follows: 


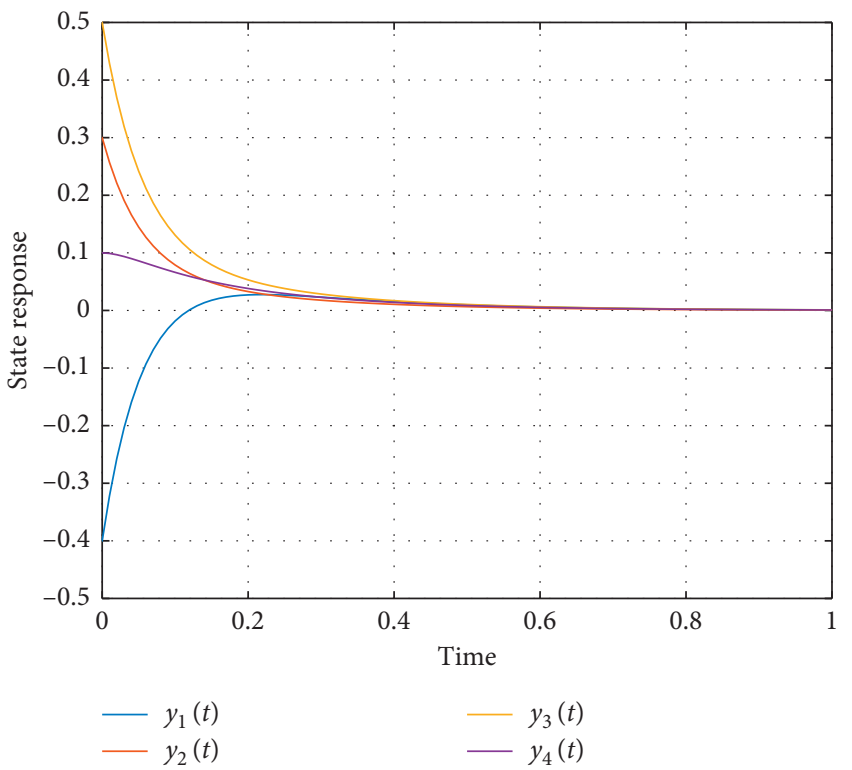

FIgURE 5: System solution for the initial state $y(0)=[-0.4,0.3,0.5,0.1]$.

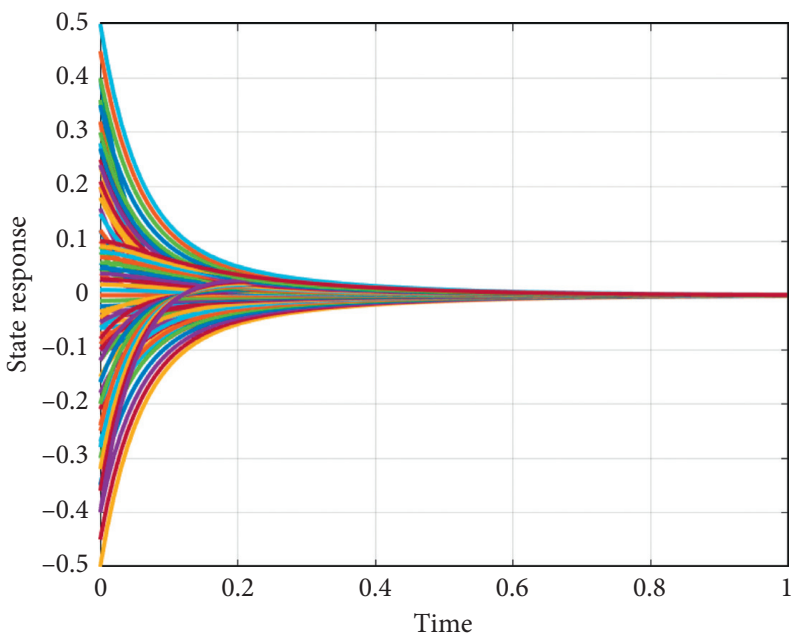

FIGURE 6: System solution for the different initial states.

$$
\begin{aligned}
\Omega_{1} & =2 \underline{\mathscr{C}} \mathscr{M} K^{-1}-\left(\mathscr{M}\left(\mathscr{D}^{*}-\Upsilon\right)+\left(\mathscr{D}^{*}-\Upsilon\right)^{T} \mathscr{M}+\left\|\mathscr{M}\left(\mathscr{D}_{*}+\Upsilon\right)+\left(\mathscr{D}_{*}+\Upsilon\right)^{T} \mathscr{M}\right\|_{2} I\right) \\
-2\left(\mathscr{M}_{\max }\|\widehat{\mathscr{R}}\|_{F}\right) I & =\left[\begin{array}{cccc}
2 c-22 & 0 & 0 & 0 \\
0 & 2 c-22 & 0 & 0 \\
0 & 0 & 2 c-22 & 0 \\
0 & 0 & 0 & 2 c-22
\end{array}\right] .
\end{aligned}
$$




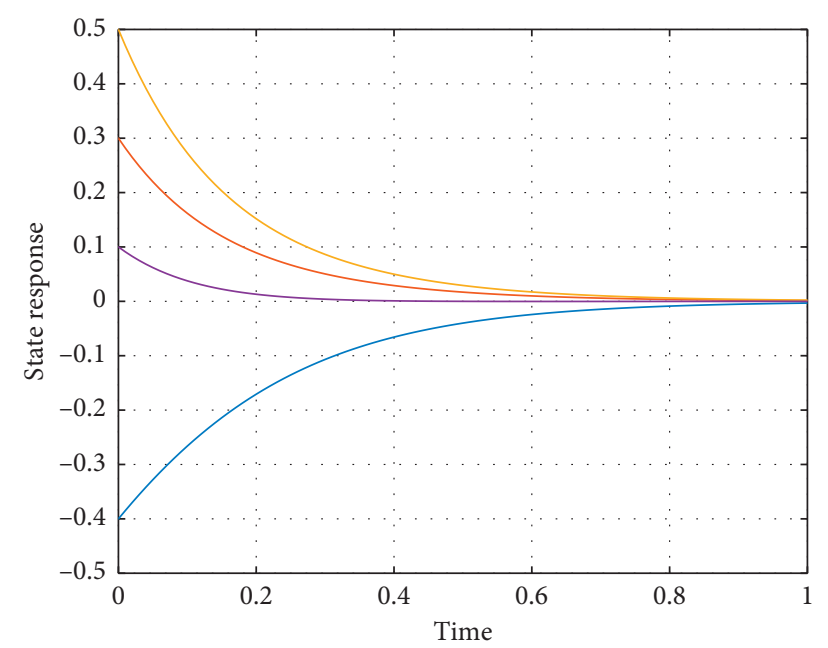

Figure 7: System solution for the initial state $y(0)=[-0.4,0.3,0.5,0.1]$.

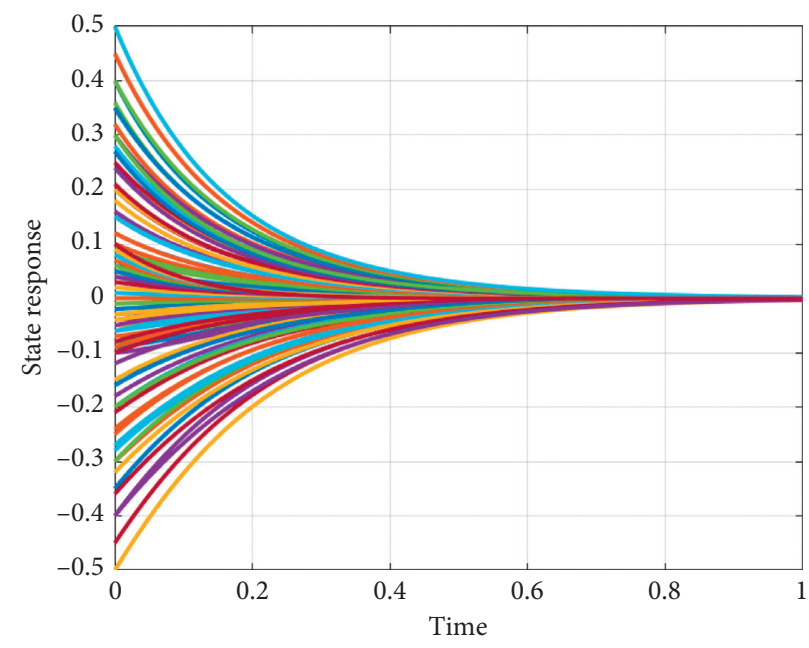

FIGURE 8: System solution for the different initial states.

$\Omega_{1}>0$, provided $c>11$. For the sufficient condition $\Omega_{1}>0$, system 2 will become GARS whenever $c>11$. Now, $\Omega_{2}$ is calculated as follows:

$$
\begin{aligned}
\Omega_{2} & =2 \underline{\mathscr{C}} \mathscr{M} K^{-1}-\left(\mathscr{M} \mathscr{D}^{*}+\left(\mathscr{D}^{*}\right)^{T} \mathscr{M}+\left\|\mathscr{M} \mathscr{D}_{*}+\left(\mathscr{D}_{*}\right)^{T} \mathscr{M}\right\|_{2} I\right)-2\left(\|\mathscr{M}\|_{2}\|\widehat{\mathscr{R}}\|_{F}\right) I \\
& =\left[\begin{array}{cccc}
2 c-20.6 & 0 & 0 & 0 \\
0 & 2 c-22.6 & 0 & 0 \\
0 & 0 & 2 c-22.6 & 0 \\
0 & 0 & 0 & 2 c-22.6
\end{array}\right] .
\end{aligned}
$$

$\Omega_{2}>0$, provided $c>11.3$. For the sufficient condition $\Omega_{2}>0$, system 2 will become GARS whenever $c>11.3$. Consider the fixed network parameters $\mathscr{C}=10 I, \mathscr{D}=$ $\overline{\mathscr{D}}$, and $\mathscr{R}=\overline{\mathscr{R}}, \tau$ is a constant, and the activation function $g(y(t))=\tanh (y(t))$. The state trajectories are depicted in Figures 5 and 6 under the initial state $y(0)=[-0.4$, $0.3,0.5,0.1]$ and for the different initial states, respectively. The activation function $g(y(t))=e^{-y^{2}(t)}-1 / e^{-y^{2}(t)}+1$, and 
the state trajectories are depicted in Figures 7 and 8 under the initial state $y(0)=[-0.4,0.3,0.5,0.1]$ and for the different initial states, respectively.

Remark 2. From Example 2, $\Omega_{2}$ is valid for $c>11.3$. Moreover, our result $\Omega_{1}$ is valid in the range $11<c<11.3$, but $\Omega_{2}$ does not hold. Therefore, we conclude that $\Omega_{1}$ is less conservative than $\Omega_{2}$ for the network parameters of this example.

From the above examples, our sufficient conditions $\Omega_{1}$ is less conservative than those imposed by the results $\Omega_{2}$ and $\Omega_{3}$. Hence, our sufficient condition is more advantageous than the previous results for the above network parameters. Our sufficient condition may have less advantage than the existing stability conditions for the different sets of network parameters. However, all such results will give sufficient conditions.

\section{Conclusion}

In this paper, global stability of NNs has been studied by using the Frobenius norm result under parameter uncertainties. Since the Frobenius norm is the easiest norm by its calculation when compared with spectral norm. By using this Frobenius norm, we have obtained a new sufficient condition for the GARS of NNs model 2. Finally, we discussed some numerical examples to illustrate the effectiveness of our result with the previous results.

\section{Data Availability}

No data were used to support this study.

\section{Conflicts of Interest}

The authors declare that they have no conflicts of interest.

\section{Acknowledgments}

The authors acknowledge King Mongkut's University of Technology Thonburi (KMUTT) and Agricultural Research Development Agency (ARDA) (PRP6205030870) for their support. The authors are very grateful to Thailand Science Research and Innovation (TSRI) (RDG6230004) for its support.

\section{References}

[1] S. Arik, "Dynamical analysis of uncertain neural networks with multiple time delays," International Journal of Systems Science, vol. 47, no. 3, pp. 730-739, 2016.

[2] S. M. A. Pahnehkolaei, A. Alfi, and J. A. T. Machado, "Delaydependent stability analysis of the QUAD vector field fractional order quaternion-valued memristive uncertain neutral type leaky integrator echo state neural networks," Neural Networks, vol. 117, pp. 307-327, 2019.

[3] S. M. A. Pahnehkolaei, A. Alfi, and J. A. T. Machado, "Stability analysis of fractional quaternion-valued leaky integrator echo state neural networks with multiple time-varying delays," Neurocomputing, vol. 331, pp. 388-402, 2019.
[4] Y. Shen and J. Wang, "Robustness analysis of global exponential stability of recurrent neural networks in the presence of time delays and random disturbances," IEEE Transactions on Neural Networks and Learning Systems, vol. 23, no. 1, pp. 87-96, 2012.

[5] N. Gunasekaran and G. Zhai, "Sampled-data state-estimation of delayed complex-valued neural networks," International Journal of Systems Science, vol. 51, no. 2, pp. 303-312, 2020.

[6] N. Gunasekaran and G. Zhai, "Stability analysis for uncertain switched delayed complex-valued neural networks," Neurocomputing, vol. 367, pp. 198-206, 2019.

[7] S. Arik, "New criteria for stability of neutral-type neural networks with multiple time delays," IEEE Transactions on Neural Networks and Learning Systems, vol. 31, no. 5, pp. 1504-1513, 2019.

[8] S. Arik, "A modified Lyapunov functional with application to stability of neutral-type neural networks with time delays," Journal of the Franklin Institute, vol. 356, no. 1, pp. 276-291, 2019.

[9] O. Faydasicok and S. Arik, "A new upper bound for the norm of interval matrices with application to robust stability analysis of delayed neural networks," Neural Networks, vol. 44, pp. 64-71, 2013.

[10] J. Cao, D.-S. Huang, and Y. Qu, "Global robust stability of delayed recurrent neural networks," Chaos, Solitons \& Fractals, vol. 23, no. 1, pp. 221-229, 2005.

[11] T. Ensari and S. Arik, "New results for robust stability of dynamical neural networks with discrete time delays," Expert Systems with Applications, vol. 37, no. 8, pp. 5925-5930, 2010.

[12] V. Singh, "Global robust stability of delayed neural networks: estimating upper limit of norm of delayed connection weight matrix," Chaos, Solitons \& Fractals, vol. 32, no. 1, pp. 259-263, 2007.

[13] M. S. Ali, N. Gunasekaran, and Q. Zhu, "State estimation of T-S fuzzy delayed neural networks with Markovian jumping parameters using sampled-data control," Fuzzy Sets and Systems, vol. 306, pp. 87-104, 2017.

[14] H. Qi, "New sufficient conditions for global robust stability of delayed neural networks," IEEE Transactions on Circuits and Systems I: Regular Papers, vol. 54, no. 5, pp. 1131-1141, 2007.

[15] O. Faydasicok and S. Arik, "Robust stability analysis of a class of neural networks with discrete time delays," Neural Networks, vol. 29-30, pp. 52-59, 2012.

[16] J.-L. Shao, T.-Z. Huang, and S. Zhou, "Some improved criteria for global robust exponential stability of neural networks with time-varying delays," Communications in Nonlinear Science and Numerical Simulation, vol. 15, no. 12, pp. 3782-3794, 2010.

[17] N. Ozcan and S. Arik, "Global robust stability analysis of neural networks with multiple time delays," IEEE Transactions on Circuits and Systems I: Regular Papers, vol. 53, no. 1, pp. 166-176, 2006.

[18] M. S. Ali, N. Gunasekaran, and M. E. Rani, "Robust stability of Hopfield delayed neural networks via an augmented L-K functional," Neurocomputing, vol. 234, pp. 198-204, 2017.

[19] N. Gunasekaran, M. S. Ali, and S. Pavithra, "Finite-time $L_{\infty}$ performance state estimation of recurrent neural networks with sampled-data signals," Neural Processing Letters, vol. 15, pp. 1379-1392, 2020.

[20] M. S. Ali and N. Gunasekaran, "State estimation of static neural networks with interval time-varying delays and sampled-data control," Computational and Applied Mathematics, vol. 37, no. 1, pp. 183-201, 2018.

[21] M. S. Ali and N. Gunasekaran, "Sampled-data state estimation of Markovian jump static neural networks with interval time- 
varying delays," Journal of Computational and Applied Mathematics, vol. 343, pp. 217-229, 2018.

[22] J. Cao and D. W. C. Ho, "A general framework for global asymptotic stability analysis of delayed neural networks based on LMI approach," Chaos, Solitons \& Fractals, vol. 24, no. 5, pp. 1317-1329, 2005.

[23] X. Liao, K.-W. Wong, Z. Wu, and G. Chen, "Novel robust stability criteria for interval delayed Hopfield neural networks," IEEE Transactions on Circuits and Systems I: Fundamental Theory and Applications, vol. 48, no. 11, pp. 1355-1359, 2001.

[24] S. Arik, "New criteria for global robust stability of delayed neural networks with norm-bounded uncertainties," IEEE Transactions on Neural Networks and Learning Systems, vol. 25, no. 6, pp. 1045-1052, 2013.

[25] X. Huang, J. Jia, Y. Fan, Z. Wang, and J. Xia, "Interval matrix method based synchronization criteria for fractional-order memristive neural networks with multiple time-varying delays," Journal of the Franklin Institute, vol. 357, no. 3, pp. 1707-1733, 2020.

[26] B. Hu, Q. Song, and Z. Zhao, "Robust state estimation for fractional-order complex-valued delayed neural networks with interval parameter uncertainties: LMI approach," Applied Mathematics and Computation, vol. 373, p. 125033, 2020.

[27] E. Yucel, M. Syed Ali, N. Gunasekaran, and S. Arik, "Sampleddata filtering of Takagi-Sugeno fuzzy neural networks with interval time-varying delays," Fuzzy Sets and Systems, vol. 316, pp. 69-81, 2017.

[28] G. Nagamani, C. Karthik, and G. Soundararajan, "Observerbased exponential stabilization for time-delay systems via augmented weighted integral inequality," Journal of the Franklin Institute, vol. 356, no. 16, pp. 9023-9042, 2019.

[29] N. Ozcan and S. Arik, "New global robust stability condition for uncertain neural networks with time delays," Neurocomputing, vol. 142, pp. 267-274, 2014.

[30] Z. Feng and J. Lam, "Stability and dissipativity analysis of distributed delay cellular neural networks," IEEE Transactions on Neural Networks, vol. 22, no. 6, pp. 976-981, 2011.

[31] C. Aouiti and E. A. Assali, "Nonlinear Lipschitz measure and adaptive control for stability and synchronization in delayed inertial Cohen Grossberg-type neural networks," International Journal of Adaptive Control and Signal Processing, vol. 33, no. 10, pp. 1457-1477, 2019.

[32] C. Aouiti, "Neutral impulsive shunting inhibitory cellular neural networks with time-varying coefficients and leakage delays," Cognitive Neurodynamics, vol. 10, no. 6, pp. 573-591, 2016.

[33] C. Aouiti, "Oscillation of impulsive neutral delay generalized high-order Hopfield neural networks," Neural Computing and Applications, vol. 29, no. 9, pp. 477-495, 2018.

[34] C. Aouiti, M. S. M'hamdi, and A. Touati, "Pseudo almost automorphic solutions of recurrent neural networks with time-varying coefficients and mixed delays," Neural Processing Letters, vol. 45, no. 1, pp. 121-140, 2017. 